Pierre-Alain Duc, Jonathan Braine and Elias Brinks, eds.

\title{
Star Formation in Virgo Intracluster Space
}

\author{
Ortwin Gerhard \\ Astronomisches Institut, Universität Basel \\ Venusstrasse 7, CH-4102 Binningen, Switzerland
}

\begin{abstract}
A number of candidate isolated compact HII regions have been discovered on combined $\mathrm{H} \alpha$, [OIII], and broadband images in the Virgo cluster. One point-like source was spectroscopically confirmed as an HII region; this object is powered by a small starburst with an estimated mass of $\sim 400 \mathrm{M}_{\odot}$ and age of $\sim 3 \mathrm{Myr}$. The object is located in the diffuse outer halo of NGC 4388, or could possibly be in intracluster space. Several resolved $\mathrm{HII}$ candidates are seen in the extended $(\sim 35 \mathrm{kpc})$ emission line region north-east of NGC 4388, perhaps triggered by the jet from the galaxy's nucleus. Star formation can thus take place far outside the main star forming regions of galaxies. The origin of the gas, the star formation, and some implications are discussed.
\end{abstract}

\section{Introduction}

Stars are usually observed to form in galaxies, that is, in disks, dwarfs, and starbursts. In some active galaxies, star formation may be triggered by energetic jet outflows (e.g., Bicknell et al. 2000). In nearby galaxy clusters, a diffuse intracluster star component has been inferred from surface brightness measurements (Bernstein et al. 1995) and detection of individual stars (e.g., Arnaboldi et al. 1996, Ferguson et al. 1998, Feldmeier 2002; see Arnaboldi, this conference). Its origin may be explained readily by dynamical processes acting on low-surface brightness disks and dwarfs, which unbind the stars from these galaxies (Moore et al. 1999), or by tidal effects on loosely bound material in infalling groups of interacting galaxies (Mihos, this conference). However, gas may also be efficiently removed from infalling galaxies by ram pressure stripping (Quilis et al. 2000, Gavazzi et al. 2001), or be tidally dissolved from them, or could fall into the cluster from outside. Recent observations show that some of this gas may form stars, contributing to the diffuse stellar component in the cluster.

\section{An isolated compact HII region in the Virgo cluster}

This object, $122543+124306$ (J2000), was found in an emission line survey for planetary nebulae in a Virgo intracluster field, using $\mathrm{H} \alpha$ and [OIII] narrow band and $\mathrm{V}+\mathrm{R}$ broad band filter photometry (Arnaboldi et al. 2003, Okamura et al. 2002), with Suprime-Cam on the Subaru Telescope. Because of the large $\mathrm{H} \alpha$ to [OIII] flux ratio the (unresolved) object was classified as a candidate compact HII region. This was confirmed with a VLT/FORS2 spectrum (Ger- 


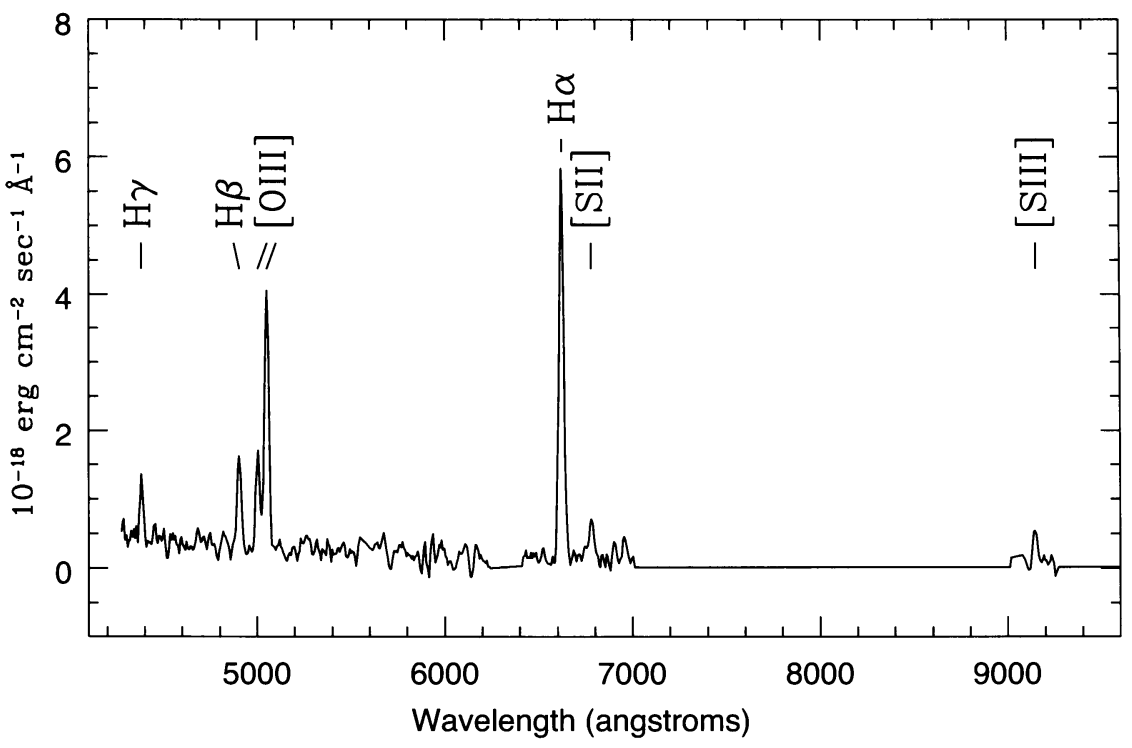

Figure 1. Observed emission spectrum of the isolated compact HII region in Virgo (from Gerhard et al. 2002).

hard et al. 2002), reproduced in Fig. 1. This clearly shows a blue continuum and a number of emission lines at Virgo redshift: $\mathrm{H} \alpha, \mathrm{H} \beta, \mathrm{H} \gamma,[\mathrm{OIII}] \lambda 5007$ and $\lambda 4959$, [SII $] \lambda \lambda 6717+6731$, and $[\mathrm{SIII}] \lambda 9069$. $\mathrm{H} \alpha$ and $[\mathrm{NII}] \lambda 6548$ and the $[\mathrm{SII}] \lambda \lambda 6717,6731$ lines are not resolved.

The reddening of the source, as estimated from the Balmer decrement, is $\mathrm{E}(\mathrm{B}-\mathrm{V})=0.40$ and hence $\mathrm{A}_{\mathrm{V}}=1.23$ mag. Using the reddening curve of Cardelli et al. (1989) and the flux calibration from the [OIII] and continuum images, the dereddened flux in the [OIII] $\lambda 5007 \AA$ line is $F_{5007}=4.0 \times 10^{-16} \mathrm{erg} \mathrm{s}^{-1} \mathrm{~cm}^{-2}$, the $\mathrm{H} \alpha$ flux is $3.9 \times 10^{-16} \mathrm{erg} \mathrm{s}^{-1} \mathrm{~cm}^{-2}$, and the dereddened V-band continuum flux from the spectrum is $F_{V}=7.0 \times 10^{-16} \mathrm{erg} \mathrm{s}^{-1} \mathrm{~cm}^{-2}$. The corresponding dereddened apparent magnitude is $\mathrm{m}_{V}=24.2$, and for a nominal Virgo distance modulus of 31.16 (at $D=17 \mathrm{Mpc}$ ) distance, Tonry et al. 2001), this gives an absolute continuum magnitude of $M_{V}=-7.0$. Thus this is a rather faint object, with total $\mathrm{V}$-band luminosity, $\mathrm{H} \alpha$ flux, and number of H-ionizing photons (Osterbrock 1989) given by $\left(d_{17}=D / 17 \mathrm{Mpc}\right)$

$$
\begin{gathered}
L_{V}=4 \pi D^{2} F_{V}=2.4 \times 10^{37} d_{17}^{2} \mathrm{erg} \mathrm{s}^{-1}, \\
L_{\mathrm{H} \alpha}=4 \pi D^{2} F_{\mathrm{H} \alpha}=1.3 \times 10^{37} d_{17}^{2} \mathrm{erg} \mathrm{s}^{-1}, \\
Q\left(\mathrm{H}^{0}\right)=2.96 L_{\mathrm{H} \alpha} / h \nu_{\mathrm{H} \alpha}=1.3 \times 10^{49} d_{17}^{2} \mathrm{~s}^{-1} \quad\left(T=10^{4} \mathrm{~K}\right) .
\end{gathered}
$$

From these numbers one can estimate the stellar mass of the ionizing stars and the age of the starburst, since the ratio $Q\left(\mathrm{H}^{0}\right) / L_{V}$ decreases rapidly with the mass of the most massive surviving O stars. Using the Starburst99 model of Leitherer et al. (1999) for metallicity 0.4 solar and a Salpeter IMF, the 
best-fitting age is 3.3 Myr. Then the luminosities in eqs. (1-3) correspond to (an average) 1-2 $\mathrm{O}$ stars and a total mass in stars of $400 \mathrm{M}_{\odot}$, i.e., a small star cluster. This cluster must have a radius smaller than that of the HII region $(\sim 3.5 \mathrm{pc}$; see Gerhard et al. 2002$)$. Clusters with these parameters have short relaxation times and dissolve by internal dynamical processes; within a few $10^{8}$ yr the stars would be added to the diffuse stellar population nearby.

The high values of $[\mathrm{OIII}] / \mathrm{H} \alpha=1.0$ and $[\mathrm{OIII}] / \mathrm{H} \beta=2.9$ in the spectrum indicate subsolar metallicity, $Z \simeq 0.4$ based on Figs. 2,3 of Dopita et al. (2000). An independent determination using the $S_{23}$ parameter calibrated by Díaz \& Pérez-Montero (2000) gives lower 0.15 to 0.25 solar, but this has additional uncertainties due to the weak [SII] line and the bright NIR sky lines, which might affect the [SIII] flux.

The inferred extinction and metallicity give an interesting estimate of the intervening hydrogen column density, using the local interstellar medium relation (Bohlin, Savage \& Drake 1978). One finds

$$
N(\mathrm{H}) \simeq 5.9 \times 10^{21} \mathrm{~cm}^{-2} \mathrm{mag}^{-1} \mathrm{E}_{B-V} Z_{\odot} / Z \sim 6 \times 10^{21} \mathrm{~cm}^{-2}
$$

If the parent cloud of the HII region is five (fifteen) times larger than the HII region itself, this corresponds to a total gas mass of some $10^{5} \mathrm{M}_{\odot}\left(10^{6} \mathrm{M}_{\odot}\right)$. Because this column density is above $10^{21} \mathrm{~cm}^{-2}$, the metallicity is $0.2-0.4$ solar, and star formation has already occurred, a fraction of this is likely to be in molecular form. Neutral HI has been detected in the halo of NGC 4388 by Vollmer \& Huchtmeier (2003); the column density near the HII region is low (van Gorkom, private communication).

\section{Induced star formation near the Virgo galaxy NGC 4388}

In a deep narrow-band $\mathrm{H} \alpha$ image, Yoshida et al. (2002) discovered a very large $(\sim 35 \mathrm{Kpc})$ extended emission line region (VEELR) to the north-east of the Seyfert 2 galaxy NGC 4388. The inferred total $\mathrm{H} \alpha$ luminosity and mass of ionized gas are $\sim 2 \times 10^{38}$ ergps and $\sim 10^{5} \mathrm{M}_{\odot}$. From the morphology of the VEELR and the excitation line ratio [OIII] $/ \mathrm{H} \alpha$ Yoshida etal. concluded that the VEELR is likely to be ionized by the radiation from the Seyfert nucleus.

In the photometry of Arnaboldi et al. (2003) of this region, a number of extended emitters in both [OIII] and $\mathrm{H} \alpha$ were found, with colors similar to the compact HII region discussed above. These are peaks in more extended emissions (see Fig. 2), and lie at distances 11-33 kpc along the direction of the VEELR identified by Yoshida et al. (2002). These objects have relatively low excitation ([OIII $] / \mathrm{H} \alpha \simeq 0.6$, in agreement with Fig. 6 of Yoshida et al. 2002), and show continuum emission near the limiting magnitude of the combined $(\mathrm{V}+\mathrm{R})$ image (Arnaboldi et al. 2003). Their half-light radii are similar in the combined continuum and in the [OIII] and $\mathrm{H} \alpha$ images, which further supports the notion that these distant sources have underlying continuum emission, and are thus ionized by OB stars rather than by the nucleus of NGC 4388. According to the discussion of Yoshida et al. (2002) this star formation could well be induced by the jet. 


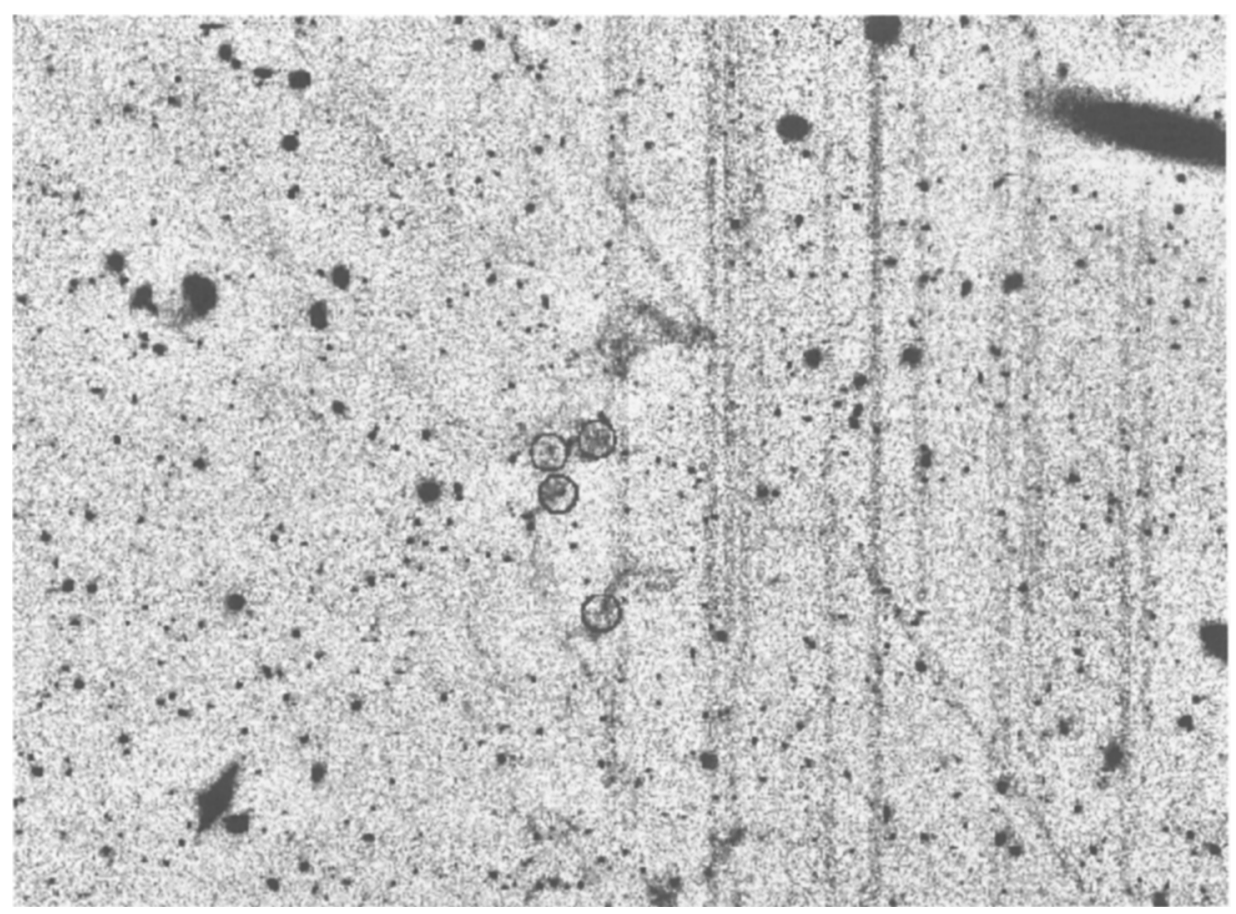

Figure 2. Some of the extended $\mathrm{H} \alpha$ and continuum emission regions, in the very extended emission line region to the NE of NGC 4388.

\section{Discussion}

\subsection{Origin of the cool gas}

Yoshida et al. (2002) discuss extensively the origin of this VEELR. They favour the scenario in which the now ionized gas is the debris of a past interaction that led to a minor merger with NGC 4388. Their arguments include the large size and streamlike morphology of the VEELR, the boxy bulge and the thickened and somewhat asymmetric disk of NGC 4388, and the similarities of these features to the results of numerical simulations of minor mergers. In an $\mathrm{H} \alpha$ survey of the A1367 cluster, Sakai et al. (2002) found a remarkable group of star-forming dwarf and HII galaxies. The properties of these objects suggest that they were formed by tidal interactions or mergers within a subgroup of the cluster. A similar interaction could have happened in the in-falling and now fast-moving NGC 4388 group some time ago; then the observed VEELR would be due to gas left over from this time.

The compact HII-region discussed above is located about 3.'4 (17 kpc projected distance) north and $0 .^{\prime} 9(4.4 \mathrm{kpc})$ west of NGC 4388 , almost perpendicular to the disk plane of this galaxy. Although it is 2.6 arcmin and $45^{\circ}$ away from the nearest part of the VEELR, it could also be associated with tidal debris. Its radial velocity inferred from the emission lines is $2670 \mathrm{~km} \mathrm{~s}^{-1}$, whereas the galaxy has $v_{r}=2520 \mathrm{~km} \mathrm{~s}^{-1}$. The near-coincidence of these numbers may indi- 
cate that the HII region is, or once was, part of the NGC 4388 system. From the large radial velocity relative to the Virgo center $\left(v_{r} \sim 1.8 \sigma_{\text {Virgo }}\right)$ and from the Tully-Fisher distance of NGC 4388 (Yasuda et al. 1997), both are probably falling through the cluster core.

Because only its projected distance from NGC 4388 is known, it is unclear whether the HII region is already unbound and moving on a different orbit in the cluster potential, or is still bound to NGC 4388. It could thus also be comparable to the high-velocity clouds in the Milky Way. Even if the HII region is currently still bound, its distance from NGC 4388 is large enough to make it vulnerable to stripping. However, Vollmer \& Huchtmeier (2003) have suggested that it might be explained as a stripped gas cloud that collapsed, decoupled from the ram pressure outflow, and is now falling back onto NGC 4388. This would explain the faster velocity of the HII region through the Virgo cluster core, as compared to NGC 4388.

\subsection{Intracluster star formation}

What has triggered the recent onset of star formation in this remote HII cloud? With its radial velocity, it will have moved $\sim 4 \mathrm{kpc}$ relative to the Virgo cluster, and $\sim 500$ pc relative to NGC 4388 , in the lifetime of the massive stars. Thus these stars will essentially have formed in situ. A possible model could be that the cloud was compressed after entering the hot intracluster medium. Bekki \& Couch (2003) have recently investigated this process with hydrodynamical simulations and conclude that such clouds would in fact be likely to make stars. The observed compact HII regions could indeed be tidally stripped or even bound gas clouds in which star formation was triggered by the ICM pressure; the newly made stars would be added to the diffuse stellar population nearby.

The current total intracluster star formation rate (SFR) estimated from 17 candidates in the field studied by Arnaboldi et al. (2003) is small, $\sim 10^{-6} \mathrm{M}_{\odot}$ $\operatorname{arcmin}^{-2} \mathrm{yr}^{-1}$ (Gerhard et al. 2002). For comparison, the intracluster luminosity inferred from planetary nebulae in this field is $\sim 10^{7} \mathrm{~L}_{B, \odot} \operatorname{arcmin}^{-2}$. However, it appears possible that at higher redshifts, when the environment of infalling galaxies was more gas-rich, formation of stars directly from cool intracluster gas could have been an important part of the origin of intracluster stars in Virgo.

The massive stars ionizing the gas in these HII regions will explode as type II supernovae and enrich the Virgo ICM with metals. From the inferred intracluster SFR, the present expected supernova rate is $\sim 10^{-8} \operatorname{arcmin}^{-2} \mathrm{yr}^{-1}$. SN II from isolated star formation could provide the enrichment inferred for the Ly $\alpha$ clouds in Virgo (Tripp et al. 2002). If such compact HII regions exist in galaxies generally, they could also be the birth places of distant B stars in the Galactic halo, some of which are too far from the disk to have been ejected from there into the halo (Conlon et al. 1992). There is no evidence for current star formation in galactic high-velocity clouds, however.

\section{Conclusion}

Star formation can occur far outside the main star forming regions in galaxies, in the diffuse outskirts of galaxies and even in intracluster space. Thus some of the observed intracluster stars in Virgo could have been made outside galaxies. 


\section{References}

Arnaboldi, M., et al. 1996, ApJ, 472, 145

Arnaboldi, M., et al. 2002, AJ, 125, 514

Bekki, K., Couch, W.J. 2003, ApJ, 596, L13

Bernstein, G.M., et al. 1995, AJ, 110, 1507

Bicknell, G.V., et al. 2000, ApJ, 540, 678

Bohlin, R.C., Savage, B.D., \& Drake, J.F. 1978, ApJ, 224, 132

Cardelli, J.A., Clayton, G.C., \& Mathis, J.S. 1989, ApJ, 345, 245

Conlon, E.S., Dufton, P.L., Keenan, F.P., McCausland, R.J.H., \& Holmgren, D. 1992, ApJ, 400, 273

Díaz, A.I., Pérez-Montero, E. 2000, MNRAS, 312, 130

Dopita, M.A., Kewley, L.J., Heisler, C.A., \& Sutherland, R.S. 2000, ApJ, 542, 224

Feldmeier, J.J. 2002, IAU Symp. 209, Intracluster Planetary Nebulae, ASP, in press, astro-ph/0201452

Ferguson, H., Tanvir, N.R., \& von Hippel, T. 1998, Nature, 391, 461

Gavazzi, G., et al. 2001, ApJ, 563, L23

Gerhard, O., Arnaboldi, M., Freeman, K.C., Okamura, S. 2002, ApJ, 580, L121

Leitherer, C., et al. 1999, ApJS, 123, 3

Moore, B., Lake, G., Quinn, T., \& Stadel, J. 1999, MNRAS, 304, 465

Okamura, S., et al. 2002, PASJ, 54, 883

Osterbrock, D.E. 1989, Astrophysics of Gaseous Nebulae and Active Galactic Nuclei, University Science Books, Mill Valley

Quilis, V., Moore, B., \& Bower, R. 2000, Science, 288, 1617

Sakai, S., Kennicutt, R.C., van der Hulst, J.M., Moss, C. 2002, ApJ, 578, 842

Tonry, J.L. et al. 2001, ApJ, 546, 681

Tripp, T.M., et al. 2002, ApJ, 575, 697

Vollmer, B., Huchtmeier, W. 203, A\&A, 406, 427

Yasuda, N., Fukugita, M., Okamura, S. 1997, ApJS, 108, 417

Yoshida, M., et al. 2002, ApJ, 567, 118 